\title{
Calcul de l'Accumulateur nécessaire pour une Installation
}

On observe fréquemment que les batteries d'accu sont insuffisantes pour remplir convenablement leur fonction et l'on se demande si les méthodes employées pour leur calcul par les ingénieurs qui les ont installées, ne sont pas exagérément approximatives.

$\mathrm{Vu}$ les prix prohibitifs actuels de ces appareils, il est indispensable de serrer de près la question. C'est le but des deux méthodes suivantes.

\section{BatTerie D'ACcu. INDÉpENDANTE}

1. Ce premier cas correspond aux hypothèses suivantes :

La batterie sera entièrement chargée avant le début de la décharge.
La figure 1 représente le courant à fournir par la batterie, en fonction de l'heure; le courant maximum est $\mathrm{I}_{\mathrm{m}}$.

La figure 2 est la figure 1 transformée en courbe monotone. On sait qu'un diagramme monotone est déduit du diagramme réel en prenant, comme abscisse correspondant à une ordonnée quelconque $i_{3}$, par exemple), une longueur égale à la somme des segments interceptés par l'horizontale $i_{3}$. Ainsi l'abscisse $i_{3} F^{\prime}$ de la figure 2 est égale à $\mathrm{AB}+\mathrm{DF}+\mathrm{EF}+\mathrm{FG}$ (quand les échelles sont les mêmes).

Il résulte de cette construction que la projection $\alpha \beta$ d'un segment 6-6' du diagramme monotone est égale à la somme des projections des segments HJ + NP interceptés sur le diagramme

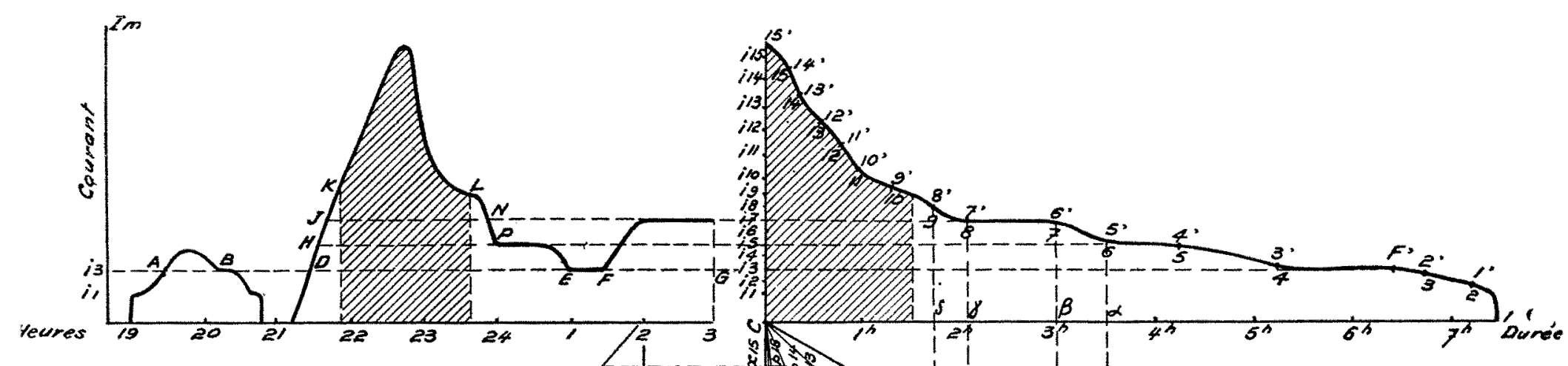

Fig. 1 Fig. 2

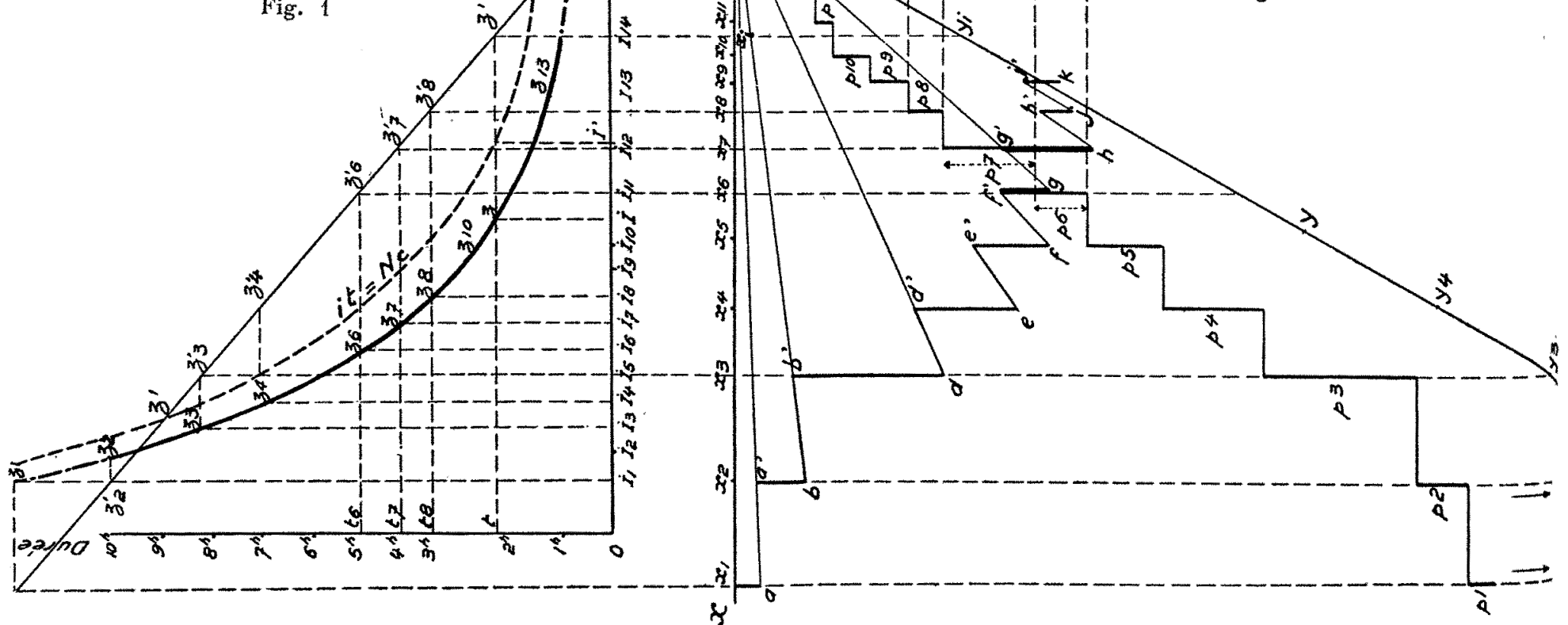

Fig. 3

Fig. 5

Elle ne recevra aucune charge pendant les interruptions momentanées de son utilisation.

Un conjoncteur-disjoncteur automatique ajoutera ou retranchera le nombre d'éléments voulu, de manière que le potentiel de la batterie, soit, à chaque instant, celui qui convient à l'appel du courant du moment. réel par les ordonnées limilant ce segment. Donc, si on déchargeait la batterie en suivan l le diagramme $1234 \ldots$... au lieu du diagramme réel de la figure 1 , on produirait chaque valeur instantanée du courant ( $i_{6}$ par excmple) pendant le même temps $d t$ dans les deux cas. Ces deux modes de décharge sont donc équivalents. En d'autres termes, le calcul d'une batterie indé 
peridante peut se faire au moyen du diagramme monotone déduit du diagramme réel de consommation.

La surface limitée par le diagramme de la figure 2 représente le nombre total d'A $\mathrm{H}$; de décharge; soit $\mathrm{N}$ ce nombre; le courant moyen est $\frac{\mathrm{N}}{7^{\mathrm{h}} 30}=\mathrm{I}$ (puisque l'abscísse maximum représente une durée de $7^{\text {h }}, 30$ ).

On commencera par choisir, dans un catalogue de fabricant d'accus, une batterie dont la capacité moyenne de décharge soit approximativement $\mathrm{N}$ pour un régime $\mathrm{I}$, et dont le courant maximum admissible par la décharge soit plus petit que $-I_{m}$.

\section{Commen't Reconnaitre si cette Batterie est suffisante}

Le catalogue du fabricant fait connaître les régimes de décharge pour les durées de 1 heure, 2 heures, 3 heures, etc.; il permet donc de construire le diagramme caractéristique de la Batterie choisie (figure 3 , trait plein).

Pour un régime quelconque de décharge $i$, la durée de décharge totale de cette batterie, complètement chargée au préalable, sera l'ordonnée " $o t$ ) du point $z$ correspondant à l'abscisse $o \mathrm{I}=i$. Si la décharge au régime $i$ ne dure qu'un temps $d t$, cette batterie aura perdu $\frac{d t}{o t}$ de sa charge totale.

Ainsi :

pour $i=i_{6}, d t_{6}=\alpha \beta$ (fig. 2), la fraction de la charge qui aura disparu pendant la décharge depuis le point représentatif 6 jusqu'au point représentatif 6 ', sera (1) :

$$
\left.\frac{d t_{6}}{o t_{6}}=\frac{\alpha \beta}{o t_{6}} \quad \text { (fig. } 2 \text { et } 3\right)
$$

quand les échelles du temps sont les mêmes dans ces deux figures. Si K est le rapport des échelles, la fraction de la charge disparue, du point 6 au point 6 , est :

$$
\frac{1}{\mathrm{~K}} \cdot \frac{\alpha \beta}{o t_{6}}
$$

Pendant que le courant aura la valeur $i_{\bar{t}}$, la fraction de charge disparue sera :

$$
\frac{1}{\mathrm{~K}} \cdot \frac{\beta \gamma}{0 t_{7}}
$$

La fraction totale disparue correspondant au segment $6-7^{\text {; }}$ est :

$$
\frac{1}{\mathrm{~K}} \cdot \frac{\alpha \beta}{o t_{6}}+\frac{1}{\mathrm{~K}} \cdot \frac{\beta y_{i}}{o t_{7}}=\frac{1}{\mathrm{~K} \cdot o t_{7}} \cdot\left(\alpha \beta \cdot \frac{o t_{7}}{o t_{6}}+\beta \gamma\right)
$$

De même la fraction totale disparue correspondant au segment $6-8$ ' est :

$$
\frac{1}{\mathrm{~K}} \cdot \frac{1}{o t_{8}}\left(\alpha \cdot \beta \frac{o t_{8}}{o t_{6}}+\frac{o t_{8}}{o t_{7}} \beta \gamma+\gamma \delta\right)
$$

Prenons (fig. 4 ci-contre) :

$$
\begin{array}{ll}
c x_{6}=\mathrm{K}^{\prime} .0 t_{6} & x_{6} y_{6}=K . t_{6} \\
c x_{7}=\mathrm{K}^{\prime} .0 t_{7} & x_{7} y_{7}=\mathrm{K} .0 t_{7} \\
c x_{8}=\mathrm{K}^{\prime} .0 t_{8} & x_{8} y_{8}=\mathrm{K} .0 t_{8} .
\end{array}
$$

portons: $\quad x_{6} a=\alpha \beta, a^{\prime} b=\beta \gamma, b^{\prime} c=\gamma \delta$

(1) On peut dire que ta déeharge, â un régime quelconque $i$, đure ici, pendant un temps $d t$ tel que $\frac{d i}{d t}$ soit le coefficient angulaire de la tangente au point correspondant de la courbe monotone.
Je dis que la fraction totale de charge disparue correspondant au segmenl $6-8$ ' est :

En effet :

$$
\frac{x_{8} c}{x_{8} y_{8}}
$$

$$
\begin{aligned}
& \frac{x_{8} a^{\prime \prime}}{x_{8} a}=\frac{x_{8} y_{8}}{x_{6} y_{6}}=\frac{o t_{8}}{o t_{6}} \quad \text { d'où : } x_{8} a^{\prime \prime}=\% ふ \beta \cdot \frac{o t_{8}}{o t_{6}} \\
& \frac{a^{\prime \prime} b^{\prime}}{a^{\prime} b}=\frac{x_{8} y_{8}}{x_{7} y_{7}}=\frac{o t_{8}}{o t_{7}} \quad \text { d'où : } a^{\prime \prime} b^{\prime}=\beta \% \cdot \frac{o t_{8}}{o t_{7}}
\end{aligned}
$$

Done :

$$
x_{8} c=x_{8} a^{\prime \prime}+a^{\prime \prime} b^{\prime}+b^{\prime} c=\alpha \beta \cdot \frac{o t_{8}}{o t_{8}}+\beta \% \cdot \frac{o t_{8}}{o t_{8}}+\gamma \delta
$$

Par conséquent :

$$
\frac{x_{8} c}{x_{8} y_{8}}=\frac{x_{8} c}{\mathrm{~K} \cdot o t_{8}}=\frac{1}{\mathrm{~K}} \cdot \frac{1}{o t_{8}}\left(\% \beta \cdot \frac{o t_{8}}{o t_{6}}+\beta \% \cdot \frac{o t_{8}}{o t_{7}}+\gamma \delta\right)
$$

Cette expression est bien identique à celle trouvée précédemment.

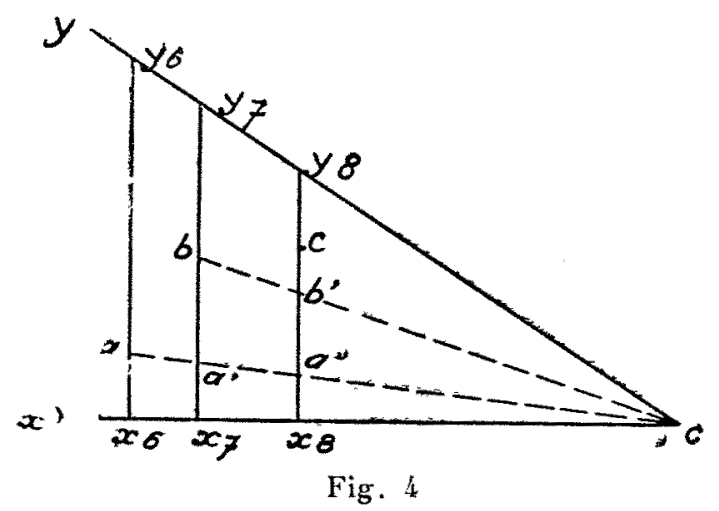

En opérant ainsi pour les projections de tous les segments, depuis 1-1' jusqu'à $15-15$ ' on obtiendra, par une série de constructions identiques à celle de la figure 4 , l'intégrale des fractions partielles de charge dépensées à chaque instant.

Si cette intégrale est plus petite que l'unité, le point terminus représentatif sera entre $c x$ et $c y$.

Si elle est plus grande que l'unité, il sera à l'extérieur de cel angle.

Dans le $1^{\mathrm{er}}$ cas l'accu choisi est trop fort.

Dans le $2^{e}$ cas, il est trop faible.

L'accu exactement suffisant correspondrait à un point reprè sentatif tombant sur $c y$.

\section{Construction dú GRAPHioue d'ESSAY.}

La construction indiquée ci-dessus donnera le graphique suivant :

Sur la figure 3, tracer une droite quelconque $z^{\prime} z^{\prime}$ (1). Prolonger les horizontales telles que $t z$ jusqu'en leur rencontre $z^{\prime}$ avec cette droite.

Reporter le point $z^{\prime}$ sur $c x$ de la figure 5 , au moyen d'une ligne de rappel $z^{\prime} x_{\mathrm{i}} ; c x_{\mathrm{i}}$ est ainsi proportionnel à $o t$.

Prendre $x_{\mathrm{i}} y_{\mathrm{i}}=o t$ à l'échelle des temps de la figure 2. Joindre $c y_{i}$ et prolonger.

On a ainsi réalisé sur la figure 5 la construction $c x_{6}, c y_{6}$ de la figure 4 .

(1) L'emplacement de cette droite a été choisi de manière que la figure reste dans les timites de la feuille. 
En faisant la mème construction pour $z_{1}, \dot{z}_{2}, \dot{z}_{3} \ldots$, on obtient les droites $x_{1} y_{1}, x_{2} y_{2}, x_{3} y_{5} \ldots$,

Sur chacune d'elles il faut projeter le segment du diagramme monotone du " $d i$ » correspondant.

Ainsi, sur $x_{6} y_{6}$ on projettera $\alpha \beta$ qui donnera $p_{6}$, sur $x_{7} y_{7}$ on projettera $\beta_{\gamma}$ qui donnera $p_{7}$, sur $x_{8} y_{8}$ on projettera $\because \delta$ qui donnera $p_{8}$.
Le point $k$ tombant en dehors de $c y$, l'accu choisi sera épuisé sans qu'on ait pu fournir les $\mathrm{AH}$ correspondant aux parties hachurées des figures 1 et 2 .

On cherchera alors un autre accu d'une capacité de décharge égale à la précédente augmentée d'une quantité au moins égale au nombre d'AH. représentés par cetle surface hachuréc. On recommencera pour ce deuxième accu la même construction.
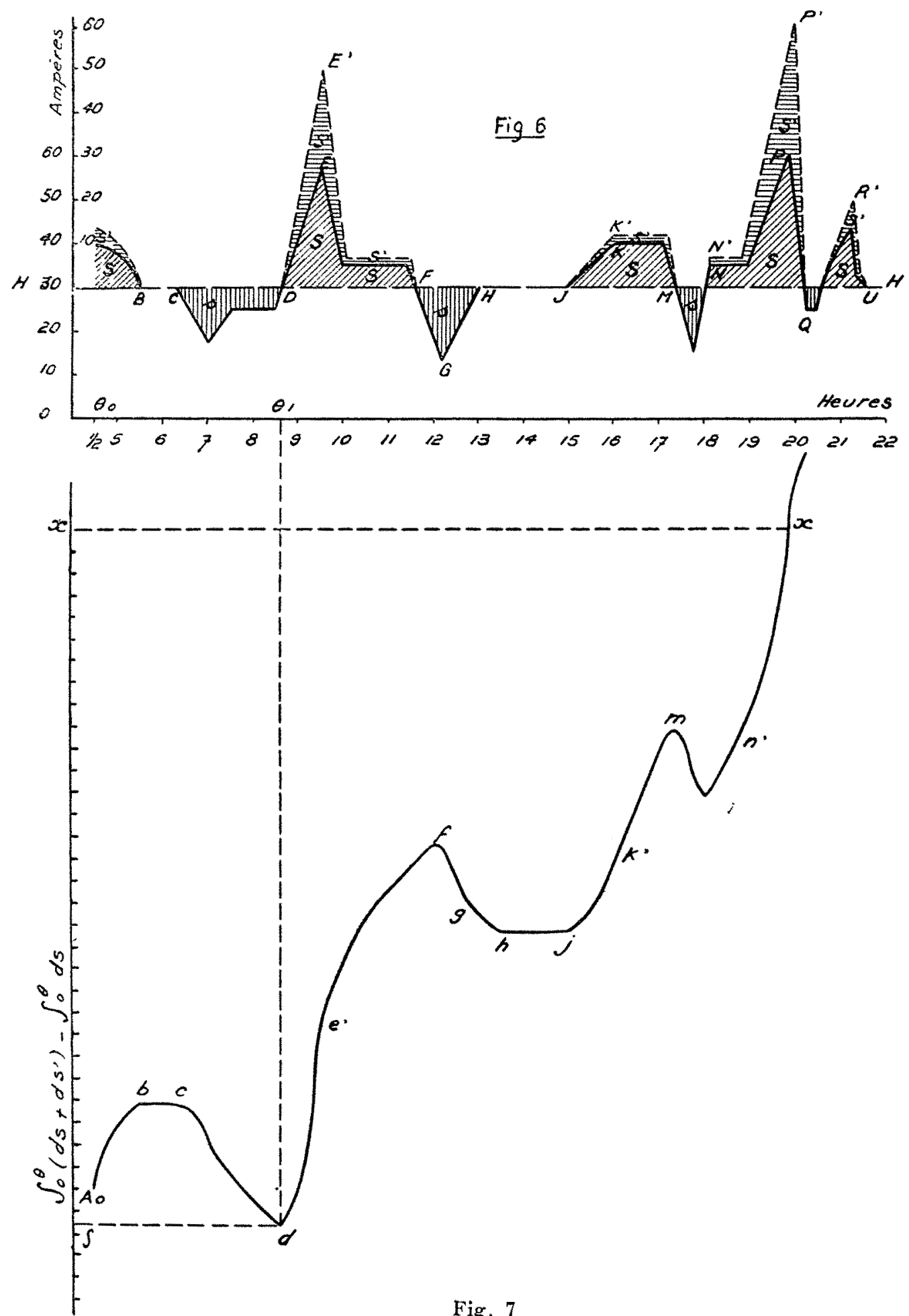

Ensuite :

porter en $x_{1} a$ la projection $p_{1}$, joindre $a c$; on obtiendra le point $a^{\text {, }}$ porter en $a^{\prime} b$ la projection $p_{2}$, joindre $b c$; on obtient ainsi le point $b$, porter en $b^{\prime} d$ la projection $p_{3}$. etc.

En continuant ainsi, on obtient les points $d^{\prime} e e^{\prime} f f^{\prime} g g^{\prime} h h^{\prime} j j^{\prime} k$.
Fig. 7

Il est vraisemblable que deux essais suffisent dans la plupart des cas.

Remarque. - Voici comment les figures 3 et 5 seront modifiées par une augmentation de capacité :

La courbe $z$ s'élève; si la feuille de papier employée est assez grande, on peut conserver la même droite $z$ ' $z$ '.

Alors chaque ordonnée $z^{\prime}$ correspond à un $x_{\mathbf{i}}$ plus grand que précédemment. $c y$ étant restée la même, les segments $p_{1}, p_{2}$ 
$p_{3} \ldots . .$. se sont donc éloignés de cette droite limite. Comme les longueurs des projections $p_{1} p_{2} p_{3} \ldots$ n'ont pas changé, le point terminus s'est avancé dans la direction de $c$.

\section{BATTERIE-TAMPON.}

1. Soit (fig. 6), le diagramme du débit journalier. L'horizontale $\mathrm{H}$ représente le débit constant des génératrices.

Les surfaces hachurées $\mathrm{S}$ au-dessus de $\mathrm{H}$, sont des périodes de décharge de la batterie; les surfaces hachurées $s$, en-dessous de H sont des périodes de charge. Par différence on aura une surface donnant une première approximation da la capacité $\mathrm{N}$ de la batterie.

On commencera par choisir dans un catalogue une batterie de capacité moyenne $\mathrm{N}$ et dont les courants maxima admissibles pour la décharge et la charge soient inférieurs aux ordonnées maxima des deux surfaces ci-dessus. Soit (fig. 3 , trait plein) sa caractéristique.

Soit $\mathrm{N}_{10}$ sa capacité de décharge en 10 heures. On peut admettre que le rendement, en $\mathrm{AH}$, est de 0,85 pour une décharge de 10 heures. La capacité de charge normale est alors $\frac{\mathrm{N}_{10}}{0,85}=\mathrm{N}_{\mathrm{c}}$.

2. Sur la figure 3, construisons l'hyperbole équilatère: $i t=\mathrm{N}_{\mathrm{c}}$.

Puisque la décharge au régima $i$ dure un temps $t$, c'est que, à ce régime, toute la matière active, formée pendant la ch rge normale complète, est décomposée en un temps $t$.

Or une charge normale complète faite en un temps $t$ exigerait un courant ' ${ }^{\prime}$ (fig. 3) tel que I' $t=N_{\mathrm{c}}$. Donc, en un temps $d t$, le courant de décharge $I$ détruit autant de matière active que n'en aurait formé I' pendant $d t$.

En d'autres termes I $d t$ perdu serait compensé par I' $d t$ gagné. Donc (fig. 6) :

$$
d_{\lrcorner} \mathrm{S}=\mathrm{I} d t \text { équivaut à } d \mathrm{~S}+d \mathrm{~S}^{\prime}=\mathrm{I}^{\prime} d t
$$

Pour connaître la charge de la batterie à un moment quelconque $\theta$, il faudra faire la somme :

$$
\text { Charge initiale }+\int_{\theta_{0}}^{\theta} d s-\int_{\theta_{0}}^{\theta}\left(d \mathrm{~S}+d \mathrm{~S}^{3}\right)
$$

c'est-à-dire remplacer la partie réelle du diagramme (fig. 6, trait continu) par un diagramma fictif (traits interrompus) dont chaque ordonnée I' est déduite de I comme il a été indiqué ci dessus au moyen du temps total $t$ de décharge au régime I (fig. 3 )

3. Traçons deux axes de coordonnées (fig. 7); portons en abscisse les temps de la figure 6 , en ordonnées les quantités

$$
\left(\int_{\theta_{0}}^{\theta}\left(d S+d S^{\prime}\right)-\int_{\theta_{0}}^{\theta} d s\right)
$$

déduites de la figure 6 .

Soit $A_{0}$ un point arbitraire représentant l'état initial; nous obtenons le diagramme $\mathrm{A}_{0} b c d e^{\prime} f g h j k$ ' $m n^{\prime}$...

Le point le plus bas étant $d$, nous serons dans les meilleures conditions d'utilisation si la charge est complète à l'heure correspondante $\theta_{1}$.

Prenons alors $a \%=\mathrm{N}_{\mathbf{c}}$.

La batterie sera entièrement déchargée à l'heure $\theta$ ñ correspondant au point de rencontre $x$ de l'horizontale $\%$ avec le diagramme $\mathrm{A}_{0} \quad b \quad c \quad d . . . . . .$. .

Pour que la batterie essayée soit la batterie optimum, il eut fallu que l'ordonnée $\chi$ fut légèrement supérieure à l'ordonnée finale $u$ (non figurée) correspondant au $p t$. $U$ de la figure 6 .
II faut alors choisir une autre batterie telle, qu'en recommençant les constructions des paragraphes 2 et 3 ci-dessus, on obtienne un point terminus satisfaisant à cette condition.

Remarque. - Si on voulait employer la méthode du $\$$ I, il faudrait, pour construire le graphique de la figure 5 , utiliser directement les projections des segments successifs du diagramme réel de consommation, sans le transformer au préalable en monotone, ce dernier ne permettant pas de suivre les modifications de l'état de la Batterie dans le temps réel.

La construction serait la suivante (fig. 8) : Déterminer, comme on l'a fait précédemment (lig. 3 et 5), sur la droite $c x$, les points $x_{1} x_{2} x_{3} \ldots$ correspondants aux valeurs du courant de la figure 6 , et la position de la droite $c y$.

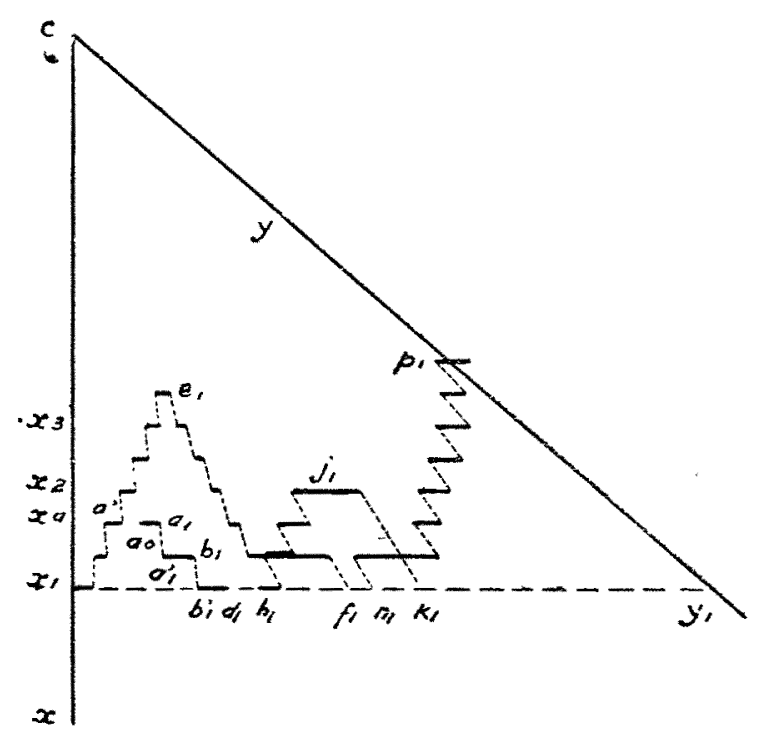

Fig. 8

Sur l'ordonnée $x_{1} y_{1}$ du courant le plus voisin de l'horizontale $\mathrm{H}$ (fig. 6), porter une longueur $x_{1} d_{1}$ telle que :

$$
\frac{x_{1} d_{1}}{x_{1} y_{1}}=\frac{s(\mathrm{BCD})}{\mathrm{N}_{\mathrm{c}}}
$$

Les segments de la partie $A B$ du diagramme de la figure 6 donnent les projections :

$$
a_{0} \quad a_{1} \quad a_{1}^{\prime} b_{1} \quad b_{1}^{\prime} d_{1} \text {. }
$$

$x_{\mathrm{a}}$ est le point correspondant au courant initial, $a_{\mathrm{o}}$ est choisi de telle sorte que la dernière projection de la portion $\mathrm{AB}$ se termine en $d_{1}$.

La première recharge parlielle $s$ (BCD) ramène de $d_{1}$ en $x_{1}$, sur $c x$, parce que la charge $\mathrm{y}$ est complète.

$x_{1} a^{\prime} e_{1} f_{1}$ correspondent à la portion DEF de la figure 6 .

Prendre cnsuite une longueur $f_{1} h_{1}$ telle que :

$$
\frac{f_{1} h_{1}}{x_{1} y_{1}}=\frac{s(\mathrm{FGH})}{\mathrm{N}_{\mathrm{c}}}
$$

La deuxième recharge partielle $s(\mathrm{FGH})$ ramène de $f_{1}$ en $h_{1}$; $h_{1} j_{1} k_{1}$ correspond à la partie H J KM.

Prendre ensuite une longueur $k_{1} n_{1}$ telle que :

$$
\frac{k_{1} n_{1}}{x_{1} y_{1}}=\frac{s(\mathrm{M})}{\mathrm{N}_{\mathrm{c}}}
$$

La troisième recharge partielle $s(\mathrm{MI})$ ramène en $n_{1} ; n_{1} p_{1}$ correspond à la portion NP de la figure 6 ; le point de rencontre avec $c y$ indique une décharge complète. 